\title{
Idiopathic Gangrene in African Children
}

\author{
A. G. G. TURPIE,* M.B., M.R.C.P.GLASG.; C. D. FORBES, * M.B., M.R.C.P., M.R.C.P.ED., M.R.C.P.GLASG \\ G. P. MCNICOL,* M.D., PH.D., M.R.C.P.ED., M.R.C.P.GLASG.
}

Brit. med. F., 1967, 3, 646-648

Gangrene of the extremities in Africans with apparently healthy arteries is a well-recognized but uncommon clinical syndrome affecting the indigenous population of East Africa (J. R. M. Miller, personal communication, 1966 ; A. A. Khan, personal communication, 1966). Both sexes and all age groups may be involved, and the condition is not confined to any particular tribal group or localized geographical area.

This communication gives an account of three African children with apparently spontaneous gangrene of the extremities.

According to current concepts it is possible that under physiological circumstances both the coagulation system and the fibrinolytic system may be constantly active to maintain an intact patent vascular tree, the coagulation system laying down a thin layer of fibrin on the vascular endothelium to seal any gaps which may occur, and the fibrinolytic system removing such fibrinous deposits after they have served their haemostatic function. In the coagulation system the inert plasma protein prothrombin is converted by thromboplastin to thrombin, which in turn brings about the conversion of the soluble protein fibrinogen to insoluble fibrin.

A similar basic structure is seen in the fibrinolytic system; plasminogen, an inactive plasma globulin, is converted by activators to plasmin, a proteolytic enzyme which under suitable circumstances digests fibrin, with release of soluble products.

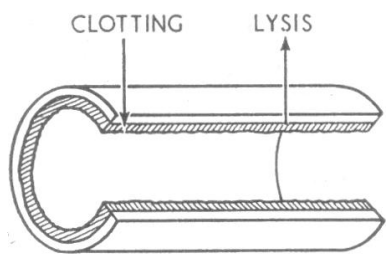
Plasminogen activators are present in many tissues, and the evidence suggests that plasminogen activator in the plasma is responsible for physiological fibrinolytic activity in the blood. A urinary activator (urokinase) probably represents, in part at least, excreted plasma activator.

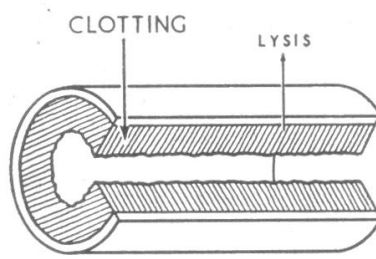

FIG. 1.-Above: the postulated dynamic equilibrium of the clotting and fibrinolytic systems. Below: how reduced fibrino lytic activity with normal cloting might be expected

In terms of the postulated dynamic equilibrium between clotting and lysis, thrombotic vascular occlusion, and hence tissue necrosis, might be expected to ensue from either hypercoagulability, with a failure of the fibrinolytic system to respond with increased activity, or from reduced fibrinolytic activity, in the presence of a normal coagulation mechanism (Fig. 1). In such circumstances reduced fibrinolytic activity might be due either to deficiency of the components of the fibrinolytic system or to the presence of inhibitors of fibrinolysis. Accordingly, in the present study particular attention was paid to the coagulation and fibrinolytic mechanisms of the affected children.

Matcrials.-Streptokinase: A. B. Kabi, Stockholm. Urokinase: purified human urokinase was supplied by Abbott

- Makerere University College, Medical School Extension, Kenyatta National Ilospital, Nairobi. On secondment from the University of Glasgow.

Requests for reprints to be sent to Dr. A. G. G. Turpie, Department of Materia Medica and Therapeutics, University Medical Unit, Stobhill General Hospital, Glasgow N.1.
Laboratories, Chicago, Illinois. Thrombin: Parke Davis thrombin topical. Thromboplastin: Rabbit-brain thromboplastin. Dade Reagents, Miami, Florida. Casein: Casein of L. Light and Company, Colnbrook, Bucks.

\section{Methods}

Plasminogen Assay.-The method of Remmert and Cohen (1949) as modified by Alkjaersig et al. (1959) was used. The method is described by McNicol and Douglas (1964).

Fibrinogen Assay.-The plasma fibrinogen levels were measured by the method of Ratnoff and Menzie (1951) as modified by Alkjaersig (1960).

One-stage "Prothrombin" Time.-This was estimated by the method described by Douglas (1962).

Thrombin Clotting-time.-The method described by Fletcher et al. (1959) was used.

Thromboplastin Generation Test.-The method described by Biggs and Douglas (1953) was used.

Plasma Recalcification Time.-The method used was that of Biggs and McFarlane (1962).

Urokinase Sensitivity Test.-This was carried out as described by $\mathrm{McNicol}$ et al. (1963). In this test the lysis time of a plasma clot in the presence of a standard amount of urokinase is measured. Results are presented in arbitrary units, based on a double logarithm plot of lysis times against units of activity, and assigning one unit of activity to a lysis time of 10 minutes. In a patient with normal fibrinogen and plasminogen levels a prolonged lysis time in this urokinase sensitivity test reflects a high level of inhibitor of fibrinolysis.

Euglobulin Lysis Time.-This test was carried out by the method described by Nilsson and Olow (1962). In this test fibrinogen, plasminogen, and plasma plasminogen activators are precipitated from the plasma ; antiplasmin is discarded with the supernatant and the precipitate is resuspended; after clotting with thrombin the lysis time is measured. As lysis times are inversely proportional to fibrinolytic activity, results are presented as suggested by Sherry et al. (1959) in arbitrary units of activity assigning to a lysis time of 300 minutes, one unit of activity. If plasminogen and fibrinogen levels are normal, variations in euglobulin lysis activity in a population can be regarded as a measure of variation in plasma plasminogen activator levels.

\section{Case 1}

A 7-year-old girl was admitted to hospital with an acute febrile illness of one day's duration. Until the onset of the illness the patient had been completely well. There was no history of drug ingestion. Clinical examination revealed a toxic febrile child with a widespread petechial rash. There was bluish discoloration of the tips of the fingers, which felt cold. The mucous membranes were pale but there was no icterus. Generalized non-tender lymphadenopathy and moderate enlargement of liver and spleen were present. The heart was normal and the blood pressure was 135/ $90 \mathrm{~mm}$. Hg. The peripheral arterial pulses were readily palpable. Digital artery pulsation was present. 
Gangrene of the fingers became evident soon after admission. Despite treatment with low-molecular-weight dextran (Rheomacrodex), corticosteroids, and penicillin, ischaemic changes appeared in the tips of the toes, and the feet later became gangrenous. Dorsalis pedis and posterior tibial pulses remained palpable in both feet. Amputation was recommended at this stage, but permission for operation was refused. Spontaneous sloughing of the gangrenous tissue of the finger-tips occurred (Fig. 2), and a clear line of demarcation developed in the feet.

The patient was discharged from hospital six weeks after the onset of the illness. She was readmitted two months later when the fore parts of the feet had sloughed ; bilateral Syme's amputation was carried out. She was discharged well after a further four months in hospital.

The most striking laboratory feature of the acute phase of the illness was autohaemagglutination. This was so pronounced that standard cross-matching of the patient's blood was impossible. Cold agglutination titre was not estimated but marked autoagglutination was noted at room temperature $\left(18.1^{\circ} \mathrm{C}\right.$.), and slight agglutinating activity was present at $37^{\circ} \mathrm{C}$. The full clinical history and an investigation of this patient have been documented by OthienoOkanga (1966). The patient's coagulation and fibrinolytic mechanisms were studied during her second admission to hospital some four months after the acute episode of the illness.

\section{Case 2}

A female child aged 2 years, with a month's history of a generalized weeping erythematous rash which had been treated unsuccessfully as scabies, was admitted to hospital with the acute onset of necrosis of the tips of her fingers and toes. There was no history of drug ingestion.

Clinical examination revealed an ill child with symmetrical peripheral gangrene of the tips of the fingers and toes. Peripheral pulses, including the digital artery pulsations, were normal. There was no lymphadenopathy and the liver and spleen were not enlarged. Neither auto-agglutination nor cold agglutination was demonstrated. The gangrenous lesiuns remained apparently uninfected and ultimately sloughed. The patient unexpectedly died after fout weeks in hospital.

At recropsy, loba: pneumonia was the only gross lesion demonstrated. Histological examination showed the vascular tree, including small arteries supplying the gangrenous tissue, to be patent. There was, however, organizing thrombus in one vein draining the gangrenoiss area.

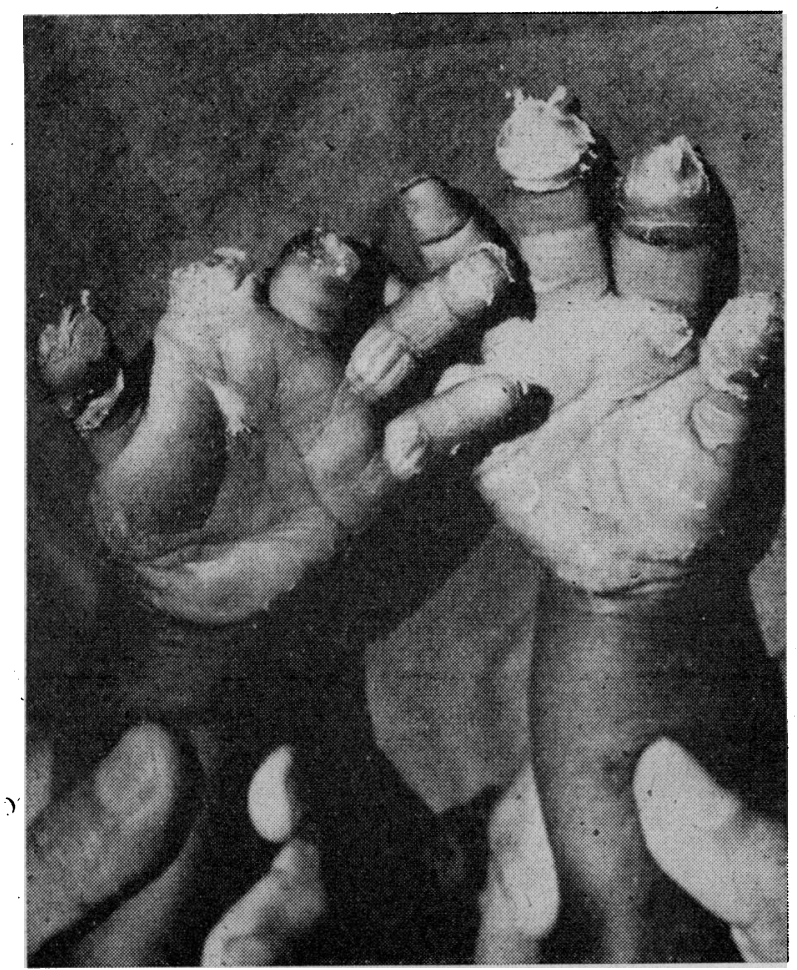

FIG. 2.-Case 1. Necrotic changes in the hands.
Studies of the fibrinolytic and coagulation mechanisms were carried out in the acute phase of the patient's disease.

\section{Case 3}

A male child aged 16 months had an illness of insidious onset of some weeks' duration. It was heralded by generalized itching, followed by the development of ulceration of the tips of his fingers. Before admission to hospital necrosis and sloughing of the tip of the little finger of the right hand developed acutely.

Apart from the gangrenous extremities clinical examination was normal. All peripheral pulses, including the digital pulses, were palpable and normal. The red blood cells showed rouleaux formation, but no agglutinins were found in the serum. Studies of the fibrinolytic system and clotting mechanisms were carried out in the acute phase of the illness.

In none of the three cases was there clinical or epidemiological evidence to suggest that they were suffering from ergot poisoning.

\section{Controls}

Plasma for control studies was obtained from age-, sex-, and tribe-matched patients from the paediatric and general me:ical wards who were suffering from a variety of miscellaneous illnesses not known to affect the haemostatic mechanism.

\section{Results}

In Fig. 3 recalcification times, thrombin clotting-times, and prothrombin times for the three patients are shown plotted against the mean and standard deviations of the values obtained from the control subjects. The plasma fibrinogen levels in the patients and the controls are shown in Fig. 4.
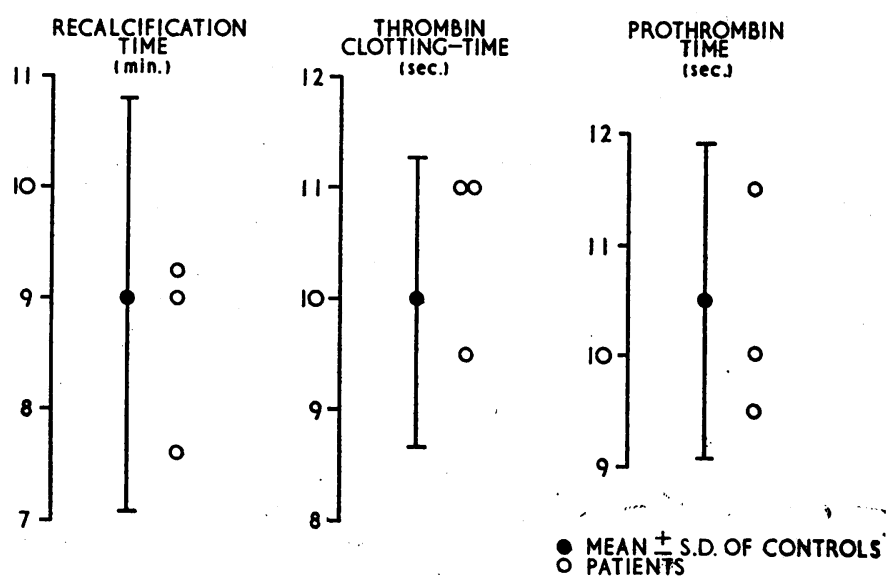

FIG. 3.-Coagulation studies performed in patients compared with the results in control subjects.

In all three patients the thromboplastin generation was normal ; one (Case 1), who had purpura, had a platelet count of 72,000/ cu.mm.- - platelet counts in Cases 2 and 3 were normal. It therefore appears that, apart from the low platelet count in Case 1 , the clotting mechanism in all three patients was normal.

Fig. 5 shows that the patients' plasminogen levels are within the range found in the controls. As plasma fibrinolytic activity is known to be low at birth and to increase over the first year or two of life (Samartzis and Cook, 1960), results for euglobulin lysis times in each patient are shown plotted separately, with controls age-matched for each patient (Fig. 6). The data in the controls confirm an increase in fibrinolytic activity with age ; and in each case activity in the patient was much depressed compared with that found in the appropriate control population.

The results of the urokinase sensitivity tests from each patient are again shown with the appropriate age-matched controls, and in the controls increased lytic activity in response to urokinase 
can be seen (Fig. 7). All three patients show greatly reduced response to urokinase as compared with their controls.

\section{Discussion}

The results presented show that in the children studied there was a significant depression of spontaneous plasma fibrinolytic activity as compared with age- and sex-matched controls, and there was also a reduction in plasma fibrinolytic activity produced in response to a standard amount of plasminogen activator. In the euglobulin lysis technique, fibrinolytic inhibitors are discarded during preparation of the euglobulin clots, and as plasminogen and fibrinogen levels were normal the results
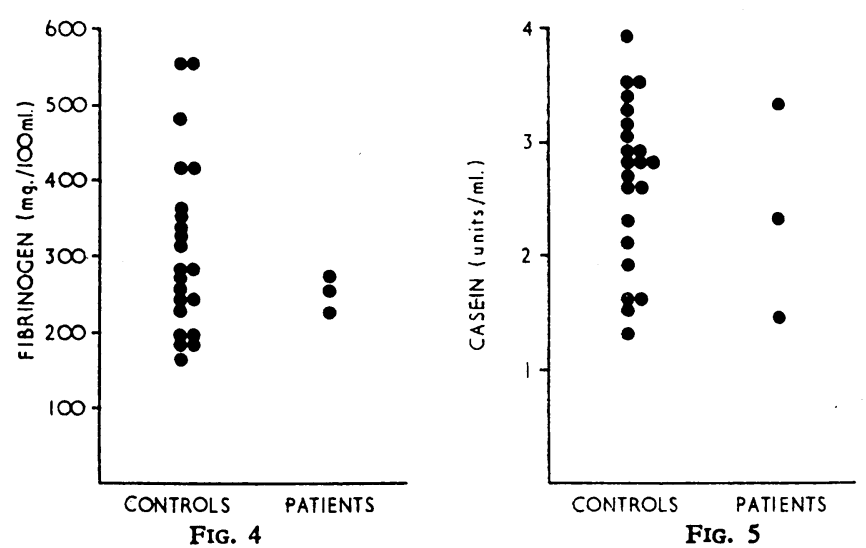

FIG. 4.-Fibrinogen levels in patients and controls. FIG. 5.-Plasminogen levels in patients and controls. Fig. 6.-Euglobulin lysis times, presented as units of activity (see text), for each patient compared with age-matched controls. FIG. 7.-Urokinase sensitivity tests for each patient compared with the results for age-matched controls. Results are presented in units (see text).

suggest that the plasma from the patients had an abnormally low level of plasminogen activator. The reduced lytic activity in response to urokinase suggests that the patients' plasmas also contained an increased amount of fibrinolytic inhibitor.

Theoretically, inhibitors of fibrinolytic activity might be expected to act at two levels-inhibition of plasminogen activation and inhibition of formed plasmin. There are at least two antiplasmins in plasma (Norman and Hill, 1958), and platelets also show antiplasmin activity. There is inferential but not conclusive evidence that plasma also possesses anti-activator properties (Paraskevas et al., 1962). The techniques used in the present study do not serve to differentiate between elevated levels of anti-activator and high antiplasmin content. No data are available as regards the basic cause or possible precipitating factors involved in this syndrome. There was no evidence of poisoning or of dysproteinaemia, nor does the condition appear to be genetically determined; an unidentified environmental factor seems the most probable explanation.

Though a causal relation between the abnormalities in fibrinolysis and the gangrene was not established, the association is of considerable theoretical and possibly also therapeutic interest. There are, so far as we can ascertain, only three reports in the literature of an increase in the levels of naturally occurring fibrinolytic inhibitor associated with severe widespread thrombotic disease (Nilsson et al., 1961; Nilsson et al., 1966 ; Brakman et al., 1966). The finding in the present patients of reduced levels of fibrinolytic activity and increased levels of fibrinolytic inhibitor, in association with tissue necrosis presumably due to thrombotic vascular occlusion, provides support for the concept of a homoeostatic function for the fibrinolytic system in vivo. It would also seem possible that a trial of therapy with fibrinolytic enzymes given early in the evolution of the disease would be worth while.

\section{Summary}

Peripheral symmetrical gangrene of unknown origin is a well-recognized but uncommon syndrome affecting all ages
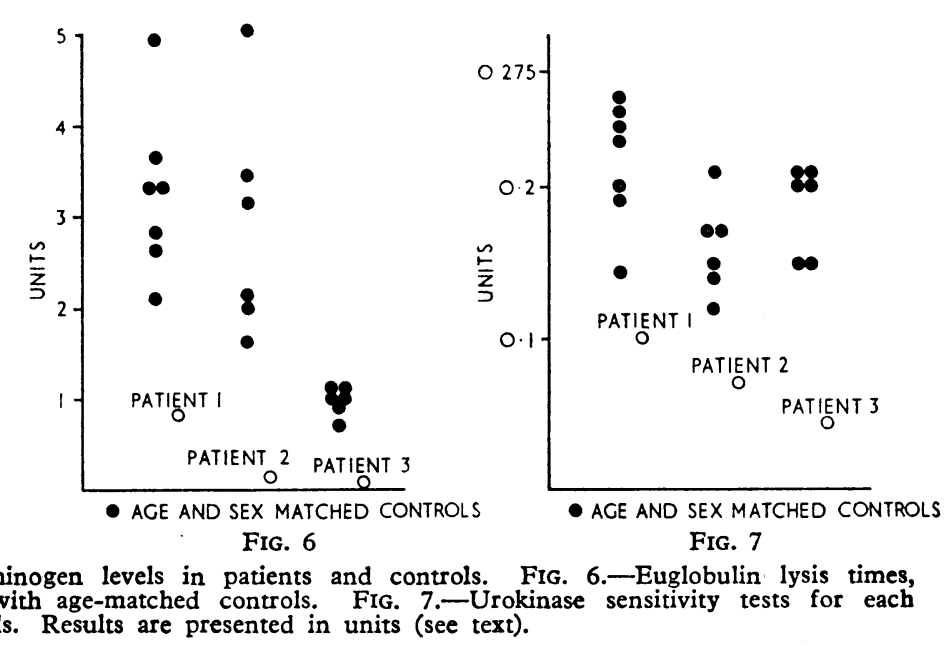

and both sexes of the indigenous population of East Africa.

The clotting and fibrinolytic mechanism of three children who suffered from this " disease" were studied. Tests of blood coagulation showed no abnormality in the patients. Of the tests of the fibrinolytic mechanism, plasminogen and fibrinogen assays were normal in each case. The euglobulin lysis time, a measure of overall spontaneous fibrinolytic activity, was grossly prolonged in each case, and the urokinase sensitivity test, a measure of plasma inhibition of fibrinolysis, was markedly reduced.

The results show that in the children studied there was a significant depression of spontaneous plasma fibrinolytic activity due in part to increased levels of fibrinolytic inhibitors.

The significance of these findings is discussed in the light of the postulated dynamic equilibrium between coagulation and fibrinolysis in the maintenance of a patent vascular tree.

We are grateful to Dr. H. P. Ojiambo and Dr. A. A. Khan for permission to study patients under their care, and to Professor $S$. Alstead and Professor A. S. Douglas for their interest in the work.

Financial assistance was provided by the Kenya Ministry of Health, East African Medical Research Council, Ministry of Overseas Development, Merck Sharp and Dohme Research Laboratories, Hoechst (East Africa) Ltd., Organon Laboratories Limited, G. D. Searle \& Co. Ltd., Boehringer Ingelheim Ltd., Pfizer Tropical Research Laboratory, The Pfizer Corporation, I.C.I. Pharmaceutical Division, and Ciba Laboratories Ltd. Reagents were provided by A. B. Kabi.

\section{REFERENCES}

Alkjaersig, N. (1960). In Conference on Thrombolytic Agents, edited by H. R. Roberts and D. Geraty, p. 316. Chapel Hill. Biggs, R., and Douglas, A. S. (1953). f. clin. Path., 6, 23.

and MacFarlane, R. G. (1962). Human Blood Coagulation and its Disorders, 3rd ed. Oxford.

Brakman, P., Mohler, E. R., Astrup, T. (1966). Scand. 7. Haemat., 3, 389.

Douglas, A. S. (1962). Anticoagulant Therapy. Oxford.

Fletcher, A. P., Alkjaersig, N., and Sherry, S. (1959). F. clin. Invest., 38, 1096.

McNicol, G. P., and Douglas, A. S. (1964). Recent Advances in Clinical Pathology. Series 4 ; edited by S. C. Dyke, p. 187. London. Pathology, B. and Douglas, A. S. (1963). Brit. med. F., 1, 909.

Nilsson, I. M., Krook, H., Sternby, N.-H., Söderberg, E., and Söderström, N.'(1961). Acta med. scand., 169, 323.

strom, N. 1961). Abid, 180, 65

- Nilehn, J.-E., Coonberg, S., Norven, G. (1966).

Norman, P. S., and Hill, B. M. (1958). F. exp. Med., 108, 639.

Norman, P. S., and Hill, B. M. (1958). F. exp. Med., 108, 639

Othieno-Okanga, J. B. (1966). East Afr. med. F., 43, 440. clin. Lab. Invest., 14, 138.

Ratnoff, O. D., Menzie, C. (1951). f. Lab. clin. Med., 37, 316.

Remmert, L. F., and Cohen, P. P. (1949). f. biol. Chem., 181, 431.

Samartzis, E. A., and Cook. C. D. (1960). Acta paediat. (Uppsala), 49,

Sherry, S., Lindemeyer, R. I., Fletcher, A. P., and Alkjaersig, N. (1959). f. clin. Invest., 38, 810 